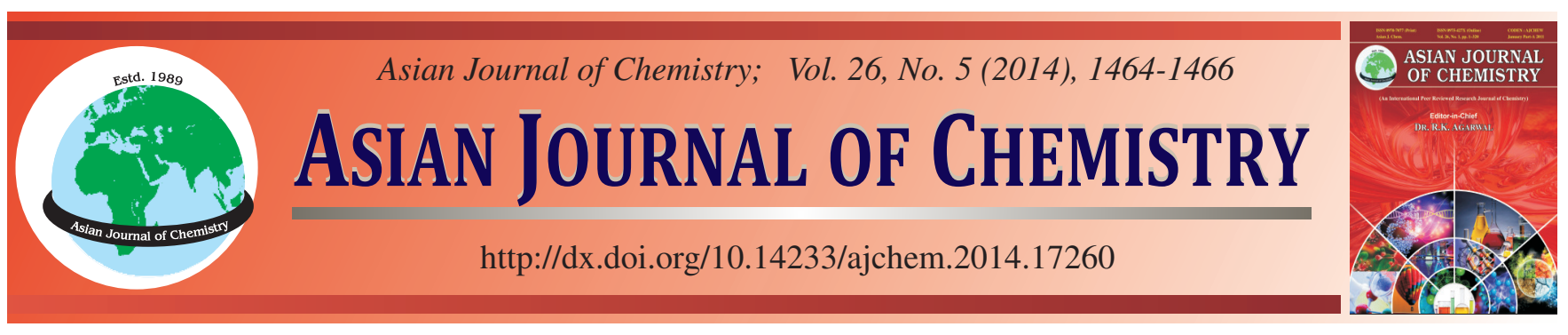

\title{
Preparation and Fluorescence Behaviour of Violet Waterborne Polyurethane using Disperse Violet 28 as Extender $\dagger$
}

\begin{abstract}
Xianhai Hu*, Jin Liu, Yu Li and Fengmin CaO
School of Materials and Chemical Engineering, Anhui University of Architecture, Hefei 230022, P.R. China

*Corresponding author: Tel/Fax: +86 551 63828100; E-mail: hxyh@ahjzu.edu.cn

A polymeric dye, waterborne polyurethane-based disperse violet 28 (WPU-DV28), was prepared by incorporation of disperse violet 28 (DV28) into waterborne polyurethane chains. The structure of waterborne polyurethane-based dye was confirmed by FT-IR and UVvisible spectra. This polymeric dye exhibited intriguing optical behaviour. WPU-DV28 latex engendered a new peaks at $490 \mathrm{~nm}$ with contrast to disperse violet 28 in UV-visible spectra. The fluorescence intensity of WPU-DV28 latex was dramatically enhanced with contrast to that of disperse violet 28 , which was attributed to hindering the formation of exciplexes in the disperse violet 28 units and augmentation of light absorption area. Furthermore, the fluorescence of WPU-DV28 was not sensitive to hydroquinone quencher.
\end{abstract}

Keywords: Polymeric dye, Waterborne polyurethane, Fluorescence enhancement, Disperse violet 28.

\section{INTRODUCTION}

Chemical anchoring of small molecular dye onto polymer matrixes to afford polymeric dye is of high interest because of the enhanced solvent resistance, resistance to migration and better processability ${ }^{1-4}$. However, most of polymeric dyes are only soluble in organic solvents and have caused severe pollution problems ${ }^{5-8}$. Waterborne polyurethane is a most versatile environment friendly material to meet the highly diversified demands of modern technologies. Therefore, the development of waterborne polyurethane-based dye is the trend in the future and have aroused much at present ${ }^{9,10}$.

In this paper, we prepared anionic water-borne polyurethane dye i.e., waterborne polyurethane-based disperse violet 28 (WPU-DV28) via a modified acetone process. Additionally, that the polymer was aqueous dispersion made it suitable for green environment. WPU-DV28 possessed the peculiar properties of optic properties, which might significantly expand the range of application in chemosensor, organic LEDs and laser active media, etc.

\section{EXPERIMENTAL}

The preparation process of WPU-DV28 was shown in Scheme-I. Poly(propylene glycol), 2,2-dimethylol propionic acid, 2,4-tolylene diisocyanate and $10 \mathrm{~mL}$ of N-methyl-2- pyrrolidone were charged into the bottom-rounded glass reactor equipped with a mechanical stirrer, a thermometer, a reflux condenser, a temperature controller according to the molar ratio. The mixture was heated at $80{ }^{\circ} \mathrm{C}$ under $\mathrm{N}_{2}$ atmosphere in presence of dibutyltin dilaurate as catalyst agent until the amount of residual $\mathrm{NCO}$ groups reached a theoretical value to produce NCO-terminated pre-polyurethane, as determined by the di-n-butylamine back-titration method. Due to the high viscosity, suitable amount of acetone was added to dilute prepolyurethane. Suitable amount of disperse violet 28 as chain extender was charged to the reactor and reacted with prepolyurethane at $80^{\circ} \mathrm{C}$ for $3 \mathrm{~h}$. Subsequently, the mixture was slowly cooled down to $30^{\circ} \mathrm{C}$; then, suitable amount of TEA was added to neutralize the carboxylic acids moieties in the DMPA. Finally, deionized water was dropwise added under vigorous stirring for $0.5 \mathrm{~h}$. After removal of the acetone by rotary vacuum evaporation under reduced pressure, WPUDV28 latex was obtained

\section{RESULTS AND DISCUSSION}

FT-IR spectra and UV-visible spectra: The FT-IR spectrum of WPU-DV28 was illustrated in Fig. 1 in comparison with that of disperse violet 28. The FT-IR spectrum of WPUDV28 showed $-\mathrm{N}-\mathrm{H},-\mathrm{C}=\mathrm{O}$ and $-\mathrm{C}-\mathrm{O}$ of $-\mathrm{NHCOO}-$ typical

†Presented at The 7th International Conference on Multi-functional Materials and Applications, held on 22-24 November 2013, Anhui University of Science \& Technology, Huainan, Anhui Province, P.R. China 


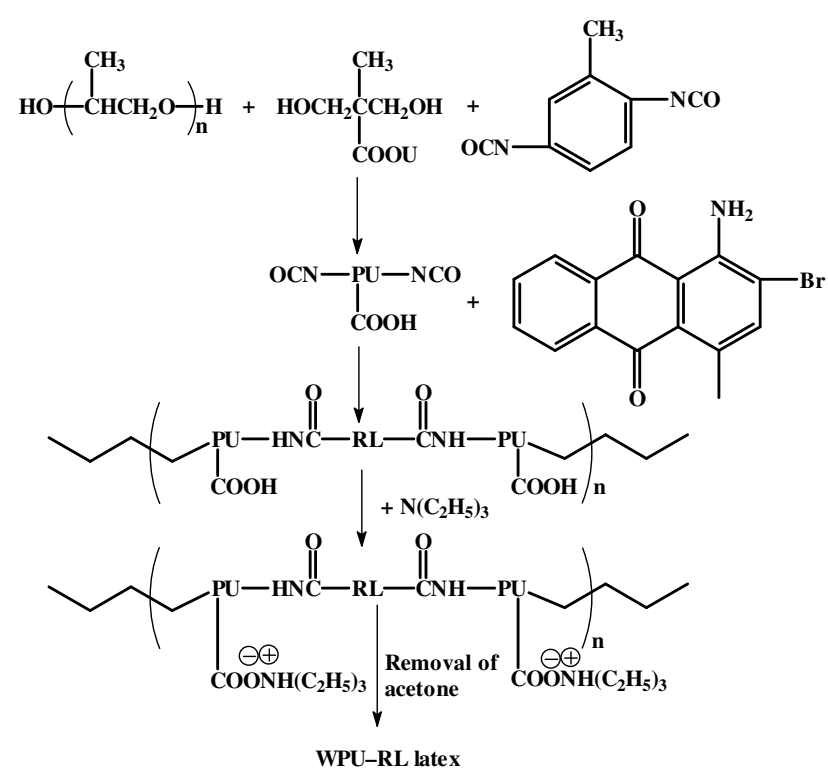

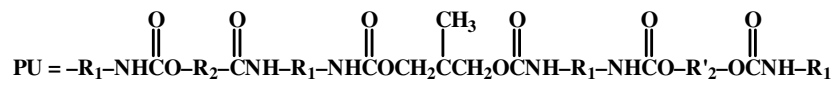

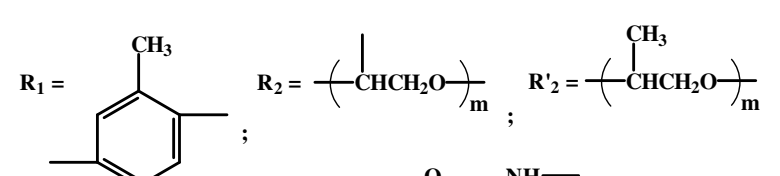<smiles>CNc1cc(Br)c(NC)c2c1C(=O)c1ccc([18O])cc1C2=O</smiles>

Scheme-I: Synthesis and structure formula of WPU-DV28

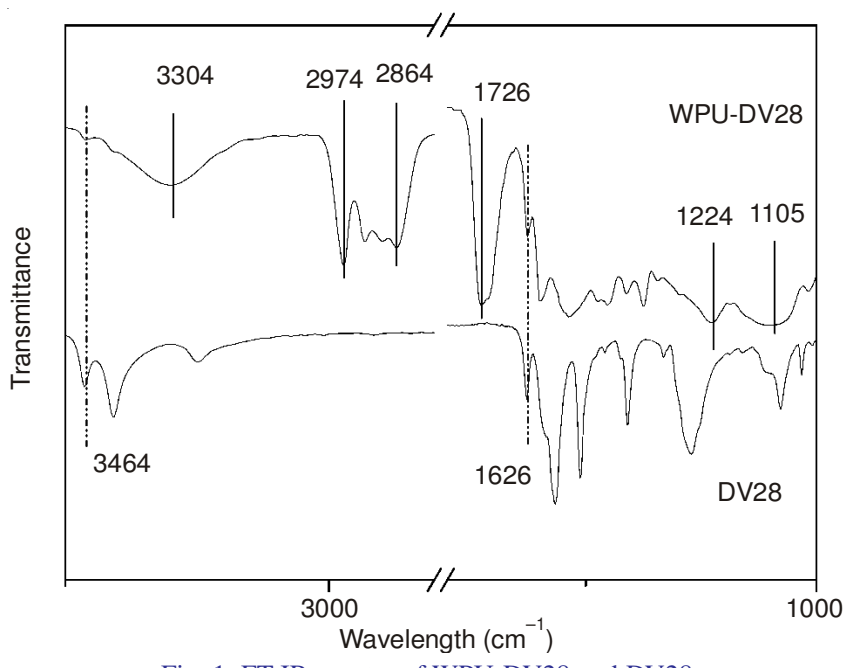

Fig. 1. FT-IR spectra of WPU-DV28 and DV28

stretching vibration at 3304,1730 and $1224 \mathrm{~cm}^{-1}$, respectively. The C-O-C in PPG and C-H of stretching vibration were observed at 1105 and $2974-2864 \mathrm{~cm}^{-1}$, respectively. Characteristic absorbance peaks corresponding to DV28 were observed distinctly at $1626 \mathrm{~cm}^{-1}[\mathrm{~V}(\mathrm{C}=\mathrm{O})]$ in anthraquinone and 3464 $\mathrm{cm}^{-1}[\mathrm{v}(\mathrm{NH})]$ of aniline structure. which supported the attachment of DV28. In addition, the absorbance peaks of WPUDV28 at around $2275 \mathrm{~cm}^{-1}$ (NCO) disappeared, which indicated the completeness of the reaction between DV28 and NCOterminated pre-polyurethane.
The UV-visible absorption spectra of WPU-DV28 latex and DV28 in acetone were shown in Fig. 2. The UV-visible spectra of WPU-DV28 showed two absorption bands associated with the anthraquinone unit at $555 \mathrm{~nm}$ and $594 \mathrm{~nm}$, which further confirmed that DV28 was attached to the polyurethane chain. WPU-DV28 latex showed a new peak corresponding to the $n-\pi^{*}$ transition at $490 \mathrm{~nm}$ compared with DV28 ${ }^{11}$.

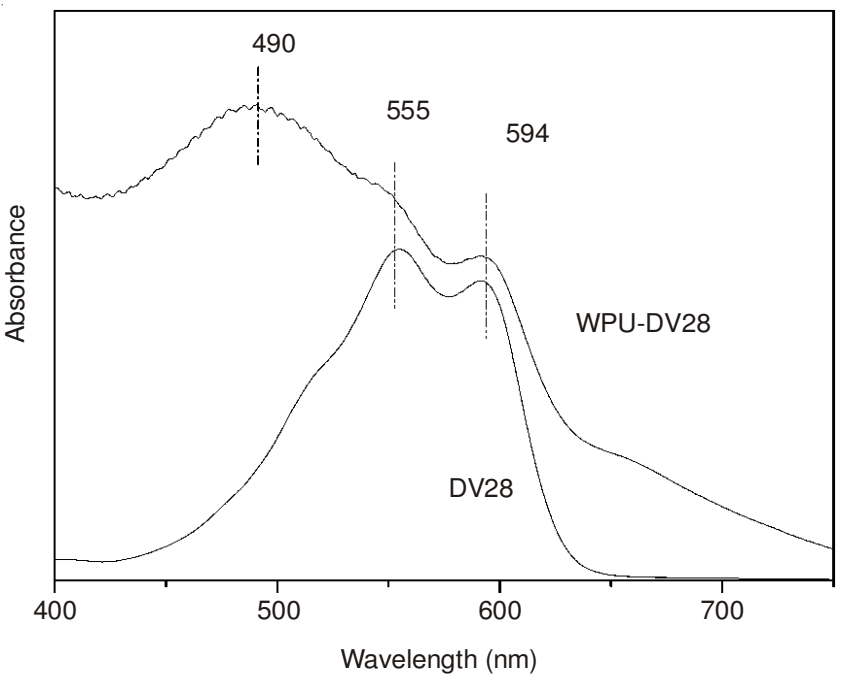

Fig. 2. UV-VIS spectra of WPU-DV28 and DV28

Fluorescence of WPU-DV28: Fluorescence spectroscopy was used to investigate the emission spectra of WPU-DV28 dispersions and DV28 in acetone as shown in Fig. 3. The fluorescence intensity of WPU-DV28 was much higher than that of DV28 in the same concentrations of chromophores. In fact, DV28 could display very faint fluorescence emission.

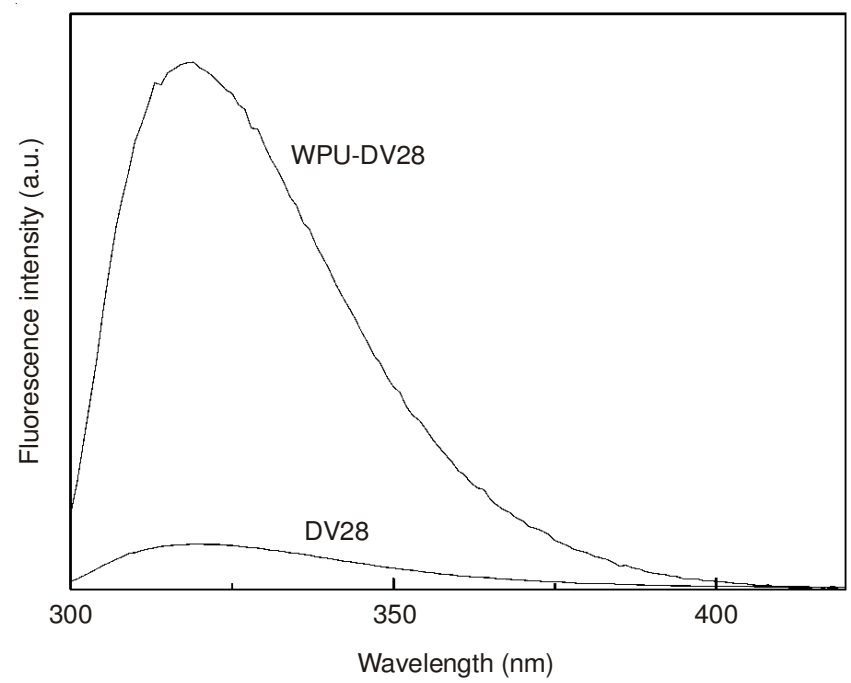

Fig. 3. Fluorescence spectra of WPU-DV28 and DV28 in acetone

The low fluorescent emission of DV28 was not due to the so-called "concentrational self-quenching effect", but because of "structural self-quenching effect". This phenomenon would be attributed to the exciplexes formation of DV28 between electron-donating amino group and electron-accepting carbonoxygen double bond that caused fluorescence quenching ${ }^{12}$. 
The fluorescence intensity of WPU-DV28 latex was greatly enhanced comparing with that of DV28, which was mainly ascribed to the following two factors: First, DV28 was fixed to the polyurethane chains, which hindered the formation of exciplex among DV28. Furthermore, the intramolecular rotation and vibration of DV28 were restricted, which had a large contribution to the radiative transition process ${ }^{13}$. Secondly, PU-DV28 formed emulsions with electrical double surface layers due to the ionization of acidic groups, leading to augmented light absorption area ${ }^{14}$.

The fluorescence spectra of WPU-DV28 under different temperatures were shown in Fig. 4. The fluorescence intensity of WPU-DV28 decreased as the temperature increased from $20-80{ }^{\circ} \mathrm{C}$, which was caused by the effect of energy transform in WPU-DV28.

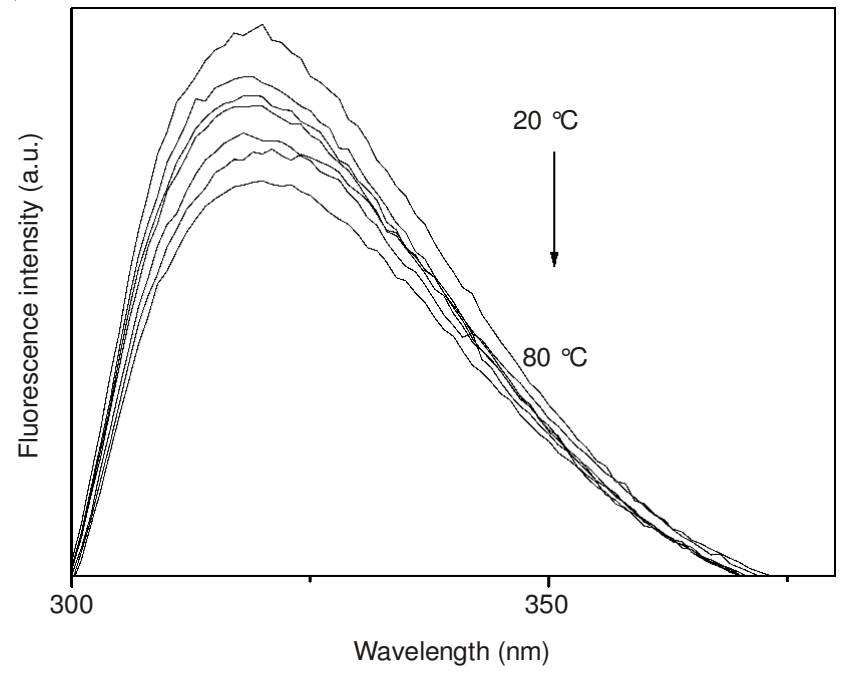

Fig. 4. Fluorescence spectra of WPU-DV28 at different temperature

The fluorescence of WPU-DV28 latex was very stable during storage. There was little distinction between the measured fluorescence spectrum for a newly prepared emulsion and for an emulsion kept at ambient temperature for 30 days. Furthermore, the fluorescence stability of WPU-DV28 latex could also be confirmed by using a fluorescence quencher such as hydroquinone. After some quenchers (for example: pyridine) were added into WPU-DV28, fluorescence decreased only a little.

\section{Conclusion}

The polymeric dye of WPU-DV28 latex was prepared via a modified acetone process. WPU-DV28 latex engendered a new peaks at $490 \mathrm{~nm}$ with contrast to DV28 in UV-VIS spectra. The fluorescence intensity of WPU-DV28 latex was dramatically enhanced with contrast to that of DV28, which was ascribed to hinder the formation of exciplexes and augment light absorption area of DV28 segments. Fluorescent intensity would decline between 20 and $80{ }^{\circ} \mathrm{C}$, mainly attributed to the effect of energy transform in WPU-DV28. The fluorescence of WPUDV28 was very stable and not sensitive to quencher.

\section{ACKNOWLEDGEMENTS}

Financial support from Natural Science Foundation of Anhui Education Department (Studies on Synthesis and Optical Performance of Waterborne Polyurethane-based Polymeric Dyes, 2014) and the Open Project of Anhui University of Architecture (Development of Insulation Materials from Cement Polyurethane-based Foams, 2012) is acknowledged.

\section{REFERENCES}

1. B.Y. Wang, X.Y. Liu, S.L. Ding and Z.X. Su, J. Polym. Res., 18, 1315 (2011).

2. B.K. Jang, S.Y. Kim and J.Y. Do, Dyes Pigments, 94, 217 (2012).

3. X.H. Hu, X.Y. Zhang, J.B. Dai and J. Liu, J. Lumin., 131, 2160 (2011).

4. X.H. Hu, X.Y. Zhang, J.B. Dai and G.W. Xu, Chin. Chem. Lett., 15, 515 (2004).

5. S. Yamaguchi and T.M. Swager, J. Am. Chem. Soc., 123, 12087 (2001).

6. CostaFerreiraPrata. A.I. Costa, L.F.V. Ferreira and J.V. Prata, J. Polym. Sci. A; Polym. Chem., 46, 6477 (2008).

7. I. Grabchev, S. Dumas and J.M. Chovelon, Polym. Adv. Technol., 19, 316 (2008).

8. I. Grabtchev, T. Konstantinov, S. Guittonneau and P. Meallier, Dyes Pigments, 35, 361 (1997).

9. J.T. Guthrie, T. Konstantinova and E. Ginova, Dyes Pigments, 34, 287 (1997).

10. F.S. Du, H. Cai, Z.C. Li and F.M. Li, J. Polym. Sci. A; Polym. Chem., 36, 1111 (1998).

11. J. Qiu, Z.-C. Li, Q.-Y. Gao, G.-Q. Yao, G.-X. Yang, J.-X. Zhang and F.-M. Li, J. Polym. Sci. A; Polym. Chem., 34, 3015 (1996).

12. H. Xianhai, X. Zhang, J. Liu and J. Dai, J. Lumin., 142, 23 (2013).

13. X.H. Hu, X.Y. Zhang and J.B. Dai, Chin. Chem. Lett., 23, 855 (2012).

14. X.H. Hu, X.Y. Zhang, J. Liu and J.B. Dai, Asian J. Chem., 25, 5503 (2013). 